\title{
Retraction Note to: Effect of Adrenaline on Cerebral Blood Oxygenation Measured by NIRS in a Rat Asphyxia Cardiac Arrest Model
}

Yu Okuma, Tsukasa Yagi, Tai Yin, Takeyuki Kiguchi, Taku Iwami, Lance B. Becker, and Koichiro Shinozaki

\section{Retraction Note to:}

Chapter 44 in: E. M. Nemoto et al. (eds.), Oxygen Transport to Tissue XLII, Advances in Experimental Medicine and Biology 1269, https://doi.org/10.1007/978-3-030-48238-1_44

The Publisher has retracted this chapter (Chapter 44, pp 277-281) as it was included in the "Tumor Oxygenation and Modeling" section of this book in error duplicating Chapter 6 (pp. 39-43) in the "Oxygen Metabolism and Health Monitoring" section. The Publisher apologises to the authors, the Editors and readers for this error. All authors agree with this retraction.

The retracted version of this chapter can be found at https://doi.org/10.1007/978-3-030-48238-1_44 